Manuscript ID

ZUMJ-1906-1290 (R2)

DOI

ORIGINAL ARTICLE

\title{
Versatility of the Pedicled Thoracodorsal Artery Perforator Flap (TDAP) Anatomic Basis and Clinical Application in breast reconstruction
}

\author{
Ahmed Attia Mohamed Attia Saleh ${ }^{(1)}$, Mohamed I Abdelhamid ${ }^{(2)}$, Mohamed Mohamed salah \\ $\operatorname{awad}^{(3)}$, Mohamed abbas hegazy elwahsh ${ }^{(3)}$ \\ General surgery resident, Zagazig University hospitals, Egypt, (2) General Surgery departments, \\ Faculty of Medicine, Zagazig University, (3) Plastic and reconstruction Surgery departments, Faculty \\ of Medicine, Zagazig University.
}

\section{Corresponding author: Ahmed Attia Mohamed Attia Saleh, M.B.B.Ch, General surgery resident, Zagazig University hospitals, Egypt E mail: ahmed.attia.saleh@gmail.com ORCID ID: https://orcid.org/0000-0002- 8281-1418

$\begin{array}{ll}\text { Submit Date } & 2019-07-02 \\ \text { Revise Date } & 2019-08-19 \\ \text { Accept Date } & 2019-09-11\end{array}$

\section{INTRODUCTION}

atissimus dorsi (LD) musculocutaneous flap has been a workhorse in soft tissue reconstruction because it is easy to dissect, reliable, and provides wide coverage for various defects ${ }^{(1)}$. Although this flap is still widely used either pedicled or as free transfers, commonly encountered restrictions, such as loss of muscle function, long-lasting seroma formation, and contour deformities at the donor site, encouraged researchers to leave the muscle

\section{ABSTRACT}

Background: the thoracodorsal artery perforator (TDAP) flap is relatively new member of perforator flap family in reconstructive surgery. TDAP flap used as pedicled flap in breast reconstruction. Advantages of the TDAP flap include homogenous flap thickness, minimal donor-site morbidity, and constant vascular anatomy with a long pedicle. Aim: to study versatility of the pedicled thoracodorsal artery perforator flap and evaluate its role in breast reconstruction. Methods: This prospective cohort study was conducted in Zagazig university Hospitals during the period from January 2018 till January 2019 on 20 patients with breast cancer who have been subjected to breast surgery and immediate breast reconstruction. Results: the mean time taken for flap elevation was $266 \pm 18$ minutes, flap elevated with single perforator in $85 \%$ of the cases and with double perforators in the remaining $15 \%$. The mean skin paddle diameters were $15.6 \pm 3.3 \mathrm{~cm}$ in length and $7.2 \pm 2.1 \mathrm{~cm}$ width. The donor side was closed by primary closure in $90 \%$ while two cases (10\%) needed grafting for closure. Objective and subjective assessment for the post procedure satisfaction showed that around $90 \%$ of the population was satisfied by the result. Conclusion: The TDAP has several advantages that make it a work horse for many soft tissue reconstructions in appropriately selected patients. The drawback of the TDAP can be manipulated by some technical modification. Keywords: thoracodorsal artery perforator flap (TDAP), Breast reconstruction, lattismaus dorsi (LD) muscle sparing flap and thoracodorsal artery (TDA).

and to harvest skin and subcutaneous tissue with the same perfusion pattern ${ }^{(2)}$.

As microsurgical skills develop and anatomic knowledge expands in flap surgery, major concerns have focused on refinements in recipient aesthetics and minimizing donor site morbidity. Perforator flap, which are described as flap consisting of skin or subcutaneous fat that receives its blood supply from isolated perforator vessels of a known source artery, 
became the most attractive option among many reconstructive surgeons ${ }^{(3)}$.

The thoracodorsal artery perforator (TDAP) flap is a fasciocutaneous flap based on a musculocutaneous perforator or perforators from the thoracodorsal vessel axis and/or its vertical branch derivative ${ }^{(4)}$.

Although this flap offers distinct advantages, including along pedicle, homogenous flap thinness, maintenance of the function of underlying $\mathrm{LD}$ has not become as popular as some other perforator flaps. This is probably because of the dissection of the TDAP flap has been described as tedious due to small diameter of the perforators and their close relation to the thoracodorsal nerve branches ${ }^{(5)}$.

Pedicled TDAP flap was reported in some clinical series for using it in axillary and breast defect and with cadaveric dissection it was shown that the arc of rotation allows shoulder, chest wall, neck, and proximal arm reconstruction in same fashion ${ }^{(6)}$. Even in the pediatric population, the use of the flap is safe due to its relatively constant vascular anatomy (7)

TDAP has a versatile utility and surgical ease of harvest and anastomosis. It has several advantages over other perforator flaps. It may well form the work horse for most soft tissue reconstructions in appropriately selected patients ${ }^{(8)}$.

The Aim of work: To study versatility of the pedicled thoracodorsal artery perforator flap and evaluate its role in breast reconstruction

\section{Patients and methods}

The Site of study: Zagazig university Hospitals during the period from January 2018 till January 2019. The work has been carried out in accordance with The Code of Ethics of the World Medical Association (Declaration of Helsinki) for studies involving humans.

Sample size: This prospective cohort study was conducted on 20 patients with breast cancer who have been subjected to breast surgery and immediate breast reconstruction.

Inclusion criteria: Patients with early stage breast cancer who are preferring mastectomy and immediate reconstruction, Patients with Modified radical mastectomy being prepared for prosthesis or with raw area between the two flaps. And fungating breast mass with large skin defect.

Exclusion criteria: Metastatic disease, patients with systemic skin disease (e.g. SclerodermaTelangiectasia), Patient older than 70 years or with severe systemic disease as cardiac, renal or patients with severe liver impairment with bleeding tendency.

Ethical Considerations: Written informed consent was obtained from all participants and the study was approved by the Faculty of medicine, Zagazig university institutional research board (IRB) (number 4371)

\section{Methods:}

- Full clinical history taking: including family history, medical disease as diabetes mellitus, heart disease, skin disease, smoking habits and history of previous operations

- Clinical examination including:

- Full general examination to exclude metastasis, skin disease, scars of previous operation and medical problems.

- Local examination for size and site (exact location of the tumor), breast skin status and presence of any breast scar, location of tumor or lumpectomy scar in relation to nipple- areola complex, state of local tissue and possible donor site and contralateral breast examination including size, contour, degree of ptosis, and shape

- All the patients were subjected to the following investigations

(a) Laboratory investigations: CBC, fasting blood sugar in diabetic patient Urea, creatinine, Prothrombin time and concentration, INR, ECG for patient $>40 \mathrm{y}$ with history of cardiac troubles. (b) Metastatic work up: Abdominal Ultrasonography, Chest X-ray and brain CT. (c) Mammography and/or breast ultrasound on both breasts. (d) Tissue diagnosis: Either cytology or tissue biopsy.

- perforator localization:

Method I: Two or three perforators are marked using these guides: 
(a) four $\mathrm{cm}$ below the inferior angle of scapula and 1-2 $\mathrm{cm}$ inside the lateral muscle border. (b) eight $\mathrm{cm}$ below the apex of the axilla and $2 \mathrm{~cm}$ medial to lateral border of latissimus dorsi muscle.

Method II:Another easier method for locating the thoracodorsal artery perforator and found it more trustable to locate the thoeacodorsal artery

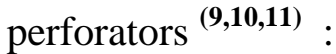

(a) for the perforator derived from the descending branch of thoracodorsal artery (a circle of a $3-\mathrm{cm}$ radius centered at a point $2 \mathrm{~cm}$ medial to the lateral border of latissimus dorsi muscle at the level of the inferior scapular angle is drawn). (b) for the perforator derived from the transverse branch of thoracodorsal artery (a circle of a $3-\mathrm{cm}$ radius centered at inferior scapular angle is drawn) ${ }^{(\mathbf{1 2})}$. Handheld Doppler can be used in two method to localise the perforator

- Evaluation of volume deficit and location fig1

- Skin paddle design: The TDAP flap was raised as a fasciocutaneous flap based on the perforators of TDA with sparing of the LD muscle and the TD nerve . the flap dimensions were fashioned according to the dimensions of soft tissue defect . the skin paddle is extended for about a $2 \mathrm{~cm}$ anterior to the free border of the LD muscle in order not to miss any direct or septocutaneous perforator arising from either the thoracodorsal artery itself or the descending branch that round the anterior border of LD muscle. the skin paddle width was determined by width of the defect and the possibility of primary closure, which was assessed by the skin-pinching test ${ }^{(\mathbf{1 3})}$. The perforator does not need to be centered to reliably perfuse this flap as ecentric position of the perforator in the flap is quite safe and ensures a long pedicle.

We use one of two designs for the skin paddle:

- Vertical skin paddle :designed along the free or lateral border of the the LD muscle this design incorporates only the perforator derived from the descending branch of the TDA

- Transverse skin paddle: designed horizontally along the posterior back to incorporate the perforators derived from both descending and transverse branches of thoracodorsal artery . That design was preferred in order to conceal the doner scar in the bra line posteriorly

- Operative technique:

a) Anesthesia: All the patient were operated under general anaesthia, broad spectrum antibiotic was given intravenously with anasthesia induction the anesthesiologest is instructed to keep the patient well perfused and not giving any vasopressors.

b) Surgical excision of mass with saftey margin and axillary lymph node clearance.

c) Patient repositioning: The patient is installed in lateral position An adhesive tape is used to stabilize the patient position by attaching that tape to the operating table passing over the patient waist. The ipsilateral shoulder is abducted 90 degree and the elbow is flexed 60 to 90 degree, the forearm is installed on a side arm.

d) Technique of TDAP flap harvest

1) the flap is incised and a monopolar diathermy is used to completely incise the dremis and subcutaneous fat and ensuring hemostasis.

2) careful dissection through the superficial fascia overlaying the LD muscle to search for any direct or septocutaneous perforator that rounds the anterior border of the LD muscle and can be used as the flap pedicle

3) Once a perforator is encountered, speeding down the dissection and using scissors instead of the monopolar diathermy to avoid spasm of the perforator, identification of the lateral (free) border of LD muscle where the posterior cautaneous branches of the lateral intrecostal nerves hooking around it dissection for this nerve branches useful If the TDAP flap is designed to be asensate one.fig2

4) Once a suitable perforator cosists of an artery accompanied by two venae comitantes and showed to be pulsating intraoperative frequent irrigation with saline is done to prevent vessel spasm and drying out of the perforator

5) Dissection and isolation of the intermuscular portion of the perforator is made with the aid of bipolar diathermy by carefully splitting the 
muscle fiber and fatty planes apart around the perforator. This step was meticulous and spent much time in flap harvesting We sometime use another technique described by $\mathrm{Kim}^{(16)}$ to make this step more easier and shorten the time of flap harvesting as whole LD muscle is preserved except of a $2 \mathrm{~cm}$ cuff around the perforator was harested and this not affect the muscle function at all .

6) Continuation of isolation and dissection of intramuscular portion of the perforator is done with perservation of the motor branches of the thoracodorsal nerve until sufficient length is gained and adequant caliber. If extra length is needed, the transverse branch of thoracodorsal artery is ligated or clipped and dissection can continue up to the origin of circumflex scapular vessels from the subscapular trunk.

7) Continuous checking the capillary refill of the skin paddle after complete detachment of the flap from the LD muscle

8) Flap transferred to the defect through a subcutaneous tunnel ensuring that that there is no twisting or pressure on the pedicle

9) The donor area was closed directly Suction drainage is usually applied for 24-48 hours If the flap width exceed $10 \mathrm{~cm}$ split thickness skin grafting was done.

10) For flap insetting the patient is placed in a supine position to compare symmetry of both breasts If an implant or expander is needed for additional volume, a pocket is created in the subpectoral plane to accommodate their placement and the flap is inset above the pectoralis muscle. After insetting, appearance and symmetry are checked with the patient in a sitting position.

11) In case of skin sparing mastectomy or in need only for volume replacement TDAP used as adipofascial flap as The flap is de-epithelialized and two breast skin flap sutured over it.

- Postoperative management: Monitoring of the flap color and warmth, prophylactic anticoagulation for 5 days postoperatively, avoidance of hypotension by I.V. fluid therapy till the patient starts adequate oral intake, prophylactic antibiotic therapy for 3 days, .monitoring of the drains and early ambulance.

- Follow up: All patients in the study were followed up for a period of 6 months post operatively for evidence of loco regional or distant failure. Longer follow up is already scheduled for them (every 2 months in the 1 st year and every 3month, in 2nd year and every 6 months after that. Mammography and sonography of the healthy breast together with sonography and MRI of the reconstructed breast to be done once per year.fig 3

- Adjuvant therapy: Postoperative adjuvant therapy was planned for most our patients and the delivery time determined to start 4 to 6 weeks postoperative to achieve maximum effect.

Statistical analysis: The collected data were summarized in terms of mean \pm Standard Deviation (SD) and range for quantitative data and frequency and percentage for qualitative data. This was carried out using STATA/SE version 11.2 for Windows (STATA Corporation, College Station, Texas).

\section{RESULTS}

The age of the study group was range from 38 to 65 years, majority of the patients were married and multiparous and almost three quarters had history of lactation. All patients desired this immediate reconstruction for moral reasons while $40 \%$ of them had added aesthetic reasons

Regarding the operative data, the mean time taken for flap elevation was $266 \pm 18$ minutes; part of this time was for dissection of the perforators with mean duration $163 \pm 28$ minutes. We succeeded to elevate the flap with single perforator in $85 \%$ of the cases and with double perforators in the remaining $15 \%$. The mean skin paddle diameters were $15.6 \pm 3.3 \mathrm{~cm}$ in length and $7.2 \pm 2.1 \mathrm{~cm}$ width. While the mean thoracodorsal pedicle length was $14.3 \pm$ $1.8 \mathrm{~cm}$. The donor side was closed by primary closure in $90 \%$ while two patients (10\%) needed grafting for closure. Eight patients (40\%) needed blood transfusion perioperative (table 1). 
With postoperative follow up single patients had partial flap necrosis, two patients had seroma either in donor or recipient side, two patients with flap congestion a single case had surgical site infections and two patients had hypertrophic scar (table 2)

Table (1): Operative data of the studied patients

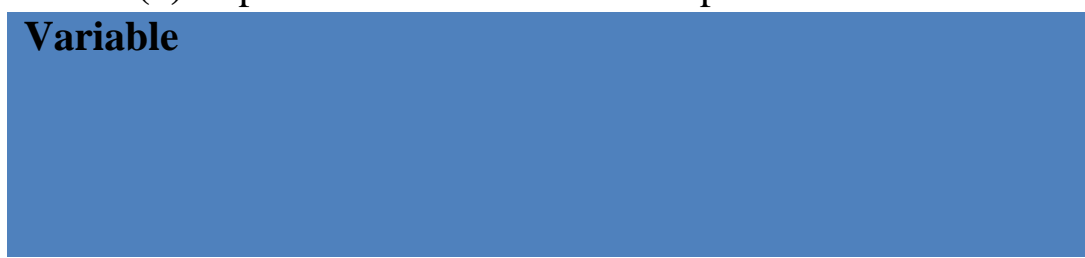

Flap harvesting operative time (min.) (Mean \pm SD)

Perforator dissection time (min.) (Mean \pm SD)

Skin paddle size $(\mathrm{cm})($ Mean \pm SD)

Length

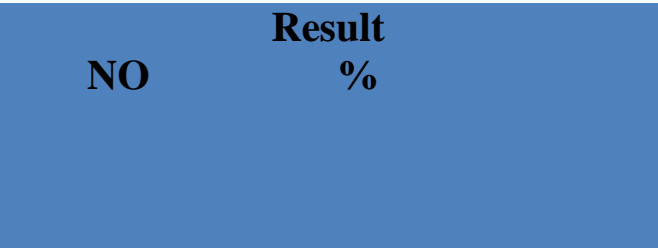

the post procedure satisfaction according to subscale using (0-2) scale for each item (general appearance, volume, contour, Inframammary fold) showed that around 90\% of the population was satisfied by the result (table 3,4).
$266.1 \pm 18.1$ range $(240-300)$

$162.6 \pm 28.3$ range $(120-210)$
$15.6 \pm 3.3$ range $(12-20)$

$7.2 \pm 2.1 \quad$ range $(5-11)$
Width

Number of included perforators

Single

3

Thoracodorsal pedicle length $(\mathrm{cm})($ Mean \pm SD)

Donor side closure

Primary

2

$(10 \%)$

$(15 \%)$

$(15 \%)$

Blood transfusion

Positive

12

$\begin{array}{ccc}14.3 \pm 1.8 & \text { range } & (10-18) \\ 18 & (90 \%) & \text { Grafting }\end{array}$

17

(85\%) Double

(10\%)

(1)

Blood transfusion

$(60 \%)$

8

(40\%) Negative

Table (2): Postoperative complications

\begin{tabular}{|l|c|c|}
\hline \multicolumn{1}{c|}{ Variable (no.=20) } & NO & $\%$ \\
\hline Partial Flap necrosis & $1 / 20$ & $(5 \%)$ \\
\hline Flap congestion & $2 / 20$ & $(10 \%)$ \\
\hline Seroma & $2 / 20$ & $(10 \%)$ \\
\hline Wound Infection & $1 / 20$ & $(5 \%)$ \\
\hline Hypertrophic scar & $2 / 20$ & $(10 \%)$ \\
\hline
\end{tabular}


Table (3): Patient satisfaction after immediate reconstruction

\begin{tabular}{|c|c|c|}
\hline Patients satisfaction (no.=20) & NO. & $\%$ \\
\hline Pleased & 12 & $(60 \%)$ \\
\hline Satisfied & 6 & $(30 \%)$ \\
\hline Unsatisfied & 2 & $(10 \%)$ \\
\hline
\end{tabular}

Table (4) Objective aesthetic assessment: the cosmetic results were assessed according to subscale using (0-2) scale for each item

\begin{tabular}{|c|c|c|c|c|}
\hline Item & $\begin{array}{c}\text { General } \\
\text { appearance }\end{array}$ & Volume & Contour & Inframammary fold \\
\hline Score & $0-2$ & $0-2$ & $0-2$ & $0-2$ \\
\hline
\end{tabular}

\begin{tabular}{|c|c|c|c|c|}
\hline Item & $\begin{array}{c}\text { Excellent } \\
\text { (Grade VI) }\end{array}$ & $\begin{array}{c}\text { Good (Grade } \\
\text { III) }\end{array}$ & $\begin{array}{c}\text { Fair (Grade } \\
\text { II) }\end{array}$ & $\begin{array}{c}\text { Poor } \\
\text { (Grade I) }\end{array}$ \\
\hline Score & $7-8$ & $6-6.9$ & $5-5.9$ & $<5$ \\
\hline Number of cases $(\%)$ & $10(50 \%)$ & $8(40 \%)$ & $2(10 \%)$ & 0 \\
\hline
\end{tabular}

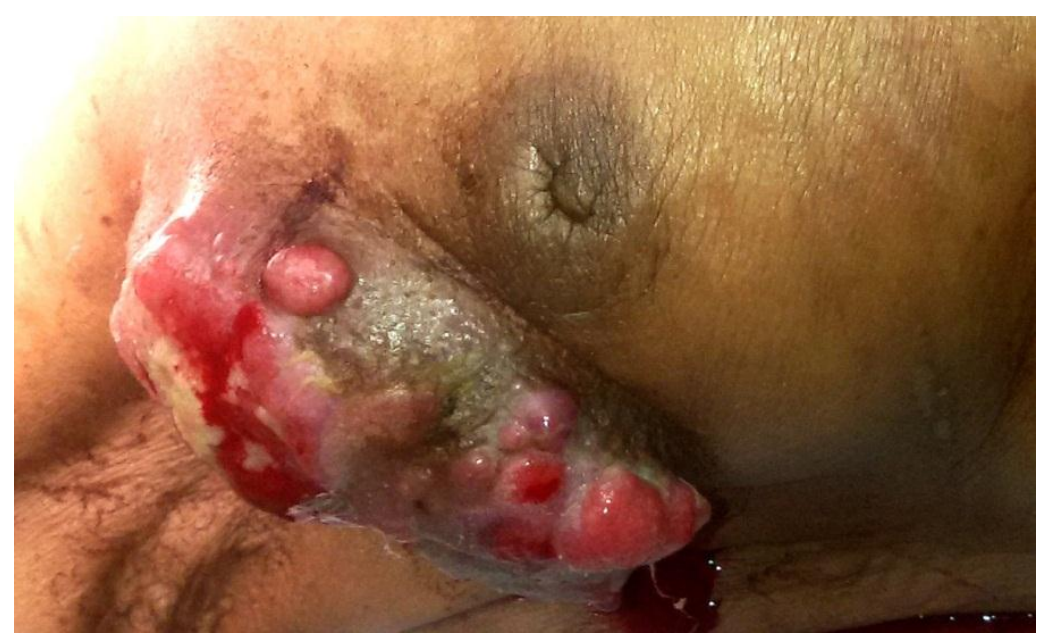

Figure ( 1 ): demonstrate the mass will be resected 


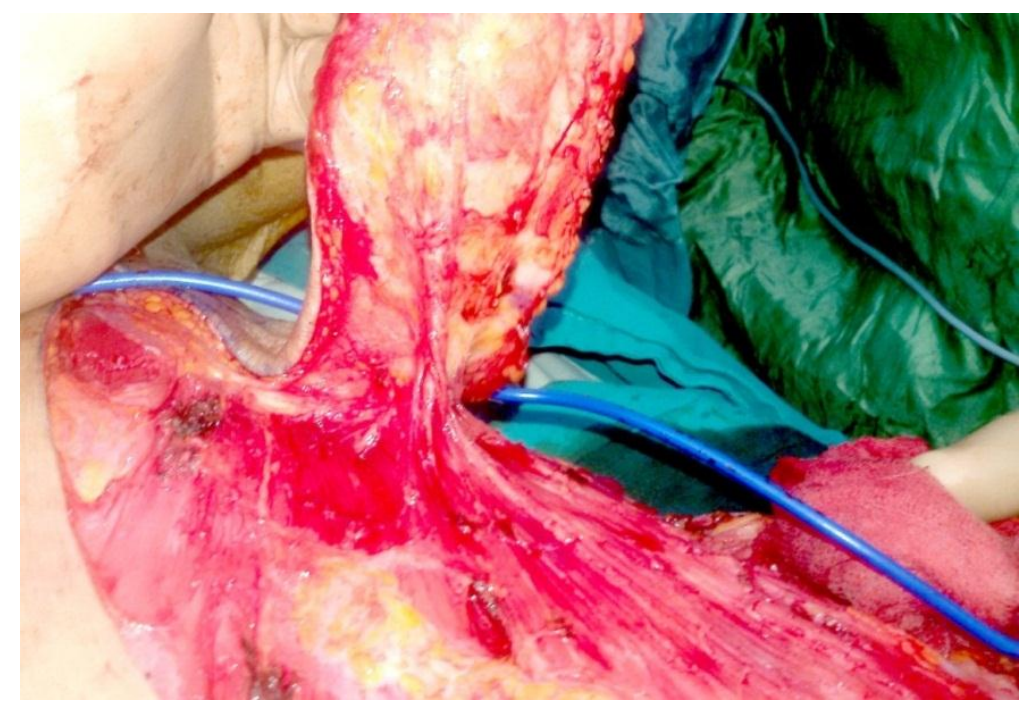

Fig 2 thoracodorsal artery perforator

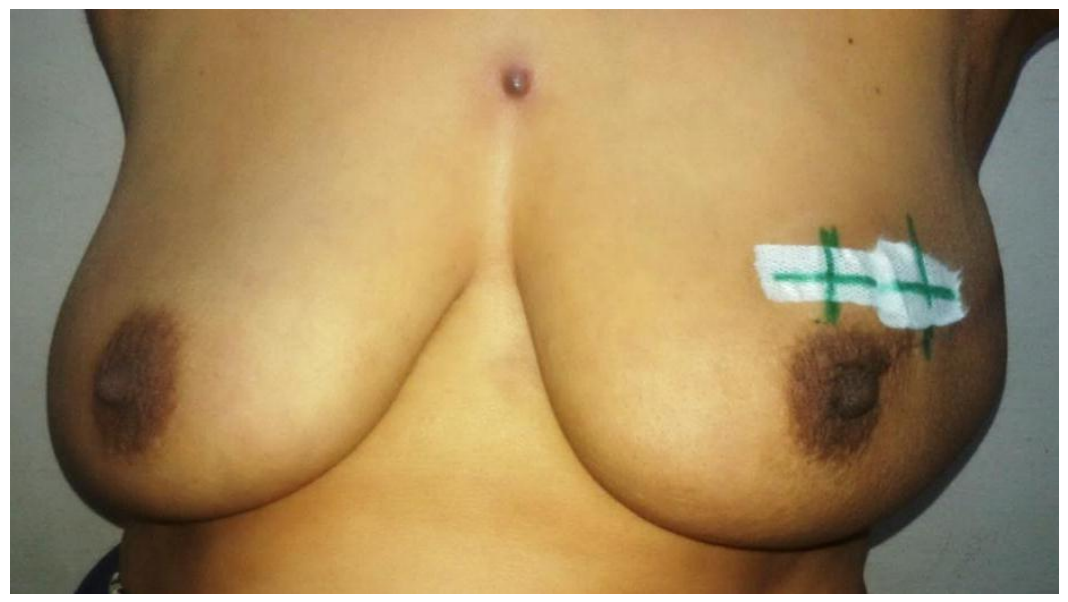

Fig 3 post operative follow up

\section{DISCUSSION}

The incorporation of the TDAP flap permits harvesting of the same skin and subcutaneous tissue area normally obtained with the conventional LD musculocutaneous flap avoiding the possible morbidities of this procedure the most voluminous part of the muscle remains under the axilla after transferring the flap to the anterior area. The muscle transferred to the breast mound is quite thin, with minimal volume contribution ${ }^{(15)}$.

Our study was on 20 patients with breast cancer who subjected to breast surgery and immediate breast reconstruction. The average age for the study group was $53.19 \pm 9.25$ years, majority of the cases were married and multiparous and almost three quarters had history of lactation. Where the breast mass was excised with safety margin and axillary dissection was undertaken. We noticed that the right breast was the affected one in $70 \%$ of cases, while the mass took more than one quadrant of the breast with positive lymph nodes in $75 \%$.

In this study, the mean time taken for flap elevation was $266 \pm 18$ minutes (range from 
240 minutes to 300 minutes); part of this time was for dissection of the perforators with mean duration $163 \pm 28$ minutes (range from 120 minutes to 210 minutes). The mean skin paddle diameters were $15.6 \pm 3.3 \mathrm{~cm}$ in length (range from 12 to $20 \mathrm{~cm}$ ) and $7.2 \pm 2.1 \mathrm{~cm}$ (range from 5 to $11 \mathrm{~cm}$ ) width. The mean thoracodorsal pedicle length was $14.3 \pm 1.8 \mathrm{~cm}$ (range from $10 \mathrm{~cm}$ to18 cm). Eight cases $(40 \%)$ needed blood transfusion perioperative. In contrast to Hamdi et al ${ }^{(16)}$, reported in their study the harvested flaps measured $23 \times 8.8 \mathrm{~cm}$ (range of 20-30 long and 8-10 cm wide). The flaps were successfully transferred with an average operative time of 190 minutes (range 135-260 minutes).

In this study the total operative time was long at start of that study due to meticulous intramuscular dissection of TDA perforator with main time $162.6 \pm 28.3$ minutes. We stated to use the technique described by Kim et al ${ }^{(14)}$ in which a muscle cuff of about 2-3 cm was harvested around the perforator after tracing of thoracodorsal pedicle to the point of entry of the perforator through the LD muscle this shorting the main time of perforator dissection.

With increased experience of flap elevation operative time decreased but other technique may offer more decrease in operative time according to Miyamoto et al (17) who state the use of the septocutaneous thoracodorsal artery perforator (TDAP-sc) flap that depend on septocutaneous perforator from the thoracodorsal vessels that has its way around the free lateral border of the LD muscle without penetrating the muscle. Therefore, the tedious intramuscular dissection unnecessary and facilitate TDAP flap harvest. This procedure can also eliminate damage to the LD muscle and the thoracodorsal nerve

We succeeded to close the donor site by primary closure in $90 \%$ while two cases $(10 \%)$ needed grafting for closure. Although the study by Sever et al ${ }^{(18)}$ state the ability to primarily close the donor site of the TDAP flap up to 12 $\mathrm{cm}$ width, we found that the maximum width that can be closed primary is $10 \mathrm{~cm}$ and this is attributed to the presence of all intact LD muscle in the field.

In our study, one case had partial flap necrosis, two cases with flap congestion that self-resolved, Seroma occurred in two cases (10\%) and was treated by aspiration 2-4 times once every 2 days, one case of wound infections and two cases had hypertrophic scar the patients were treated with local measures. By the way when Judkins and singletary ${ }^{(19)}$ study the advantages, disadvantages and results that can be expected after immediate breast reconstruction utilizing TDAP flap in 100 patients with an average length of follow up was 20 months (range 8 to 44 months) state that ; The major complications were rare (1\% partial necrosis and $1 \%$ total necrosis). The minor complications were represented mainly with dorsal seroma and was the main drawback of the technique and occurred in $79 \%$ specially in obese patients. Donor site morbidity in terms of wound infection and scarring was relatively low and occurred in $4 \%$ of cases.

Regarding our patients satisfaction Twelve patients $(60 \%)$ were pleased with their new breasts. Six patients $(30 \%)$ were satisfied with the reconstruction. Two patients (10\%) were unsatisfied, Excellent results were observed in ten cases $(50 \%)$. Good results were observed in eight cases (40\%). Fair results were observed in two cases $(10 \%)$. No poor result was reported after reconstruction. On the other hand Judkins and singletary ${ }^{(19)}$ reported that the level of patient satisfaction was high $87 \%$ of the patients were deeply satisfied, $10 \%$ were satisfied and only $3 \%$ were poorly satisfied. The aesthetic results have been judged excellent by surgeons in $85 \%$, good in $12 \%$ and poor in $3 \%$ and no results judged bad.

According to Amin et al (20) overall complication rate was $20 \%$ with transient flap congestion being the most common complication(7.5\%). This is a rather peculiar complication for perforator flaps in general and have recorded no donor site morbidities, in particular zero percent seromas after using this technique This is probablydue to the lack of 
dead space resulting from muscle conservation. This is a major advantage for the TDAP technique

The main drawback found during our work, was the time need for meticulous intramuscular dissection of the TDA preforator through the LD muscle. Also, the mismatch of handheld Doppler mapping and the intraoperative finding of the perforator. But, nevertheless a perforator can be found. This flap is indeed versatile in designing of the skin flap based on perforator localisation without the fear of encroaching a different anatomical area or significantly affecting any landmark and we advise to good to perforator topographe

The main advantages of TDAP : The presence of perforator is consistent, a large skin paddle can be harvested on a single perforator with preservation the LD muscle and TD nerve the incidence of post-operative donor site seroma is decreased and shoulder function is preserved, The TDAP flap donor site is well hidden and concealed. If the flap is vertically oriented the scar is hidden under the arm and appears only on arm elevation. If the flap is transversely oriented the scar is hidden in brassiere line posteriorly, the skin paddle can be fashioned in any way (vertically, obliquely and transversely) over the latissimus dorsi muscle. This facilitates tailoring of skin paddle according to the defect, it has a long vascular pedicle this make flap transfer an insetting more easily, The TDAP flap contains no muscle allowing more reconstructive precision in longterm follow up. No contour change due to muscle atrophy that occurs with LD muscle or myocutaneous flap because of muscle denervation

Thoracodorsal artery perforator flap represents a valid and safe option, which adds to the reconstructive oncoplastic surgeons. This technique combines the advantages of perforator and the advantages of pedicled flaps, Thus, it can achieve an acceptable cosmetic outcome with minimal donor site morbidity ${ }^{(20)}$. In this study we add an advantage to the use of the TDAP flap for volume fullness in a cavity of the breast after its evacuation from tumor through using TDAP as adipofascial flap.

Conclusion: The versatility of TDAP has several advantages that make it a workhorse flap for most breast reconstructions requiring soft tissue cover. The drawback of the TDAP can be manipulated by some technical modification.

Conflict of interest: The authors declare no conflict of interest.

Funding sources: The authors have no funding to report

\section{Acknowledgement}

The authors are grateful for the patients without whom this study would not have been done.

\section{REFERENCES}

1) Lassen M, Krag C, Nielsen IM. Thr latissimus dorsi flap: an overview. Scandinavian journal of plastic and reconstructive surgery. 1985; 19(1):41-51.

2) Titely O, Spryou G, Fatah M. Preventing seroma in latissimus dorsi flap donor site. British journal of plastic surgery. 1997; 50(2):106-8.

3) Blondeel PN, Van Landuty KH, Monstery SJ, Hzmdi M, Matton GE, Allen RJ, et al. The "Gent" consensus on perforator flap terminology: Preliminary definitions. Plastic and reconstructive surgery. 2003;112(5):1378-83

4) Hayakawa TJ. Thoracodorsal Artery Perforator flap (TAP flap). Rudolf Buntic, Rudy Buntic, MD.2017.

5) Ayhan S, Tuncer S, Demir Y, Kandal S. Thoracodorsal artery perforator flap: a versatile alternative for varies soft tissue defects. Journal of reconstructive microsurgery. 2008;24(04):285-93

6) Hamdi M, Van Landuyt K, Monstery S, Blondeel P. Pedicled perforator flap in breast reconstruction : a new concept. British journal of plastic surgery. 2004; 57(60:531-9.

7) Kim SE, Rhyou IH, Suh BG, Chung KC. Use of thoracodorsal artery perforator flap for soft tissue reconstruction in children. Annals of plastic surgery. 2006; 56(4):451-4.

8) Jain L, Kumta SM, Purohit SK, Raut R. Thoracodorsal artery perforator flap: indeed a versatile flap. Indian journal of plastic surgery: official publication of the Association of Plastic Surgeon of India. 2015; 48(2):153.

9) Angrigiani M, Rancati A, Escudero E, Artero G, Extended thoracodorsal artery perforator flap for breast reconstruction. Gland surgery. 2015; 4(6):519.

10)Guerra AB, Metzinger SE, Lund KM, Cooper MM, Allen RJ, Dupin CL. The thoracodorsal artery perforator flap: Clinical experience and anatomic study with emphasis on harvest 
techniques. Plastic and reconstructive surgery. 2004; 114(1):32-41.

11)Heitman C, Guerra A, Metzinger SW, Levin LS, Allen RJ. The thoracodorsal artery perforator flap: Anatomic basis and clinical application. Annals of plastic surgery. 2003; 51(1):23-9.

12)Mun G-H, Lee S-J, Jeon B-J. Perforator topography of the thoracodorsal artery perforator flap. Plastic and reconstructive surgery. 2008;121(2):497-504

13)Kim SW, Youn DG, Kim JT, Kim YH. A thoracodorsal artery perforator chimeric free flap for prevention of microvascular pedicle compression in lower extremity reconstruction. Microsurgery. 2015.

14)Kim SW, Youn S, Kim JT, Kim YH. A modified method for harvesting thoracodorsal artery perforator flaps in a simple and time-saving approach. Microsurgery. 2016:36(8):642-6

15)Angrigiani M, Rancati A, Escudero E, Artero G, Extended thoracodorsal artery perforator flap for breast reconstruction. Gland surgery. 2015; 4(6):519.
16)Hamdi S , Barone $\mathbf{C}$ and Adesi V. Use of the Thoracodorsal Artery Perforator (TDAP) Flap With Implant in Breast Reconstruction Annals of Plastic Surgery.2008; Issue: Volume 61(2): 143-146.

17)Miyamoto, S., Arikawa, M., Kagaya, Y., \& Fukunaga, Y. Septocutaneous thoracodorsal artery perforator flaps: a retrospective cohort study. Journal of Plastic, Reconstructive \& Aesthetic Surgery. 2019; 72(1), 78-84.

18)Sever, C., Uygur, F., Kulahci, Y., Karagoz, H., \& Sahin, C. Thoracodorsal artery perforator fasciocutaneous flap: a versatile alternative for coverage of various soft tissue defects. Indian Journal of Plastic Surgery: Official Publication of the Association of Plastic Surgeons of India.2012; 45(3), 478.

19)Judkins A F and singletany $\mathbf{S}$ E. Surveillance studies and long term follow up care. NewYork: springer- verlag; 233. 2009.

20)Amin, A. A., Rifaat, M., Farahat, A., \& Hashem, T.The role of thoracodorsal artery perforator flap in oncoplastic breast surgery. Journal of the Egyptian National Cancer Institute 2017 ; 29(2), 83-87

\section{HOW TO CITE}

attia mohamed attia, A., Ibrahim Abdelhamid mansy, M., hegazy elwahsh, M., Awad, M. Versatility of the Pedicled Thoracodorsal Artery Perforator Flap (TDAP) Anatomic Basis and Clinical Application in breast reconstruction. Zagazig University Medical Journal, 2021; 2(228-237): -. doi: 10.21608/zumj.2019.13814.1290 